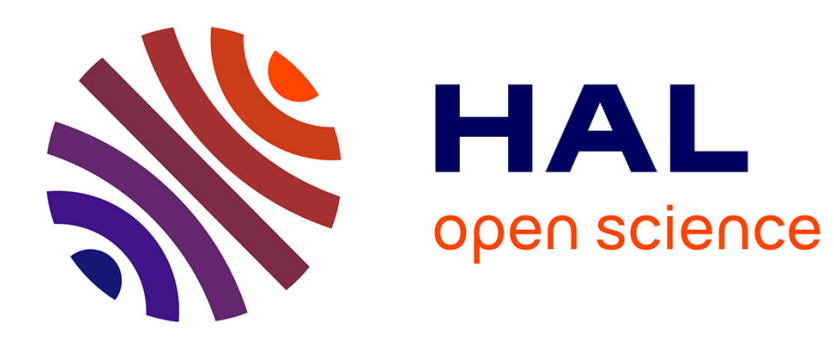

\title{
6-dimensional properties of Al0.86Mn0.14 alloy
}

P.A. Kalugin, A. Yu. Kitayev, L.S. Levitov

\section{To cite this version:}

P.A. Kalugin, A. Yu. Kitayev, L.S. Levitov. 6-dimensional properties of Al0.86Mn0.14 alloy. Journal de Physique Lettres, 1985, 46 (13), pp.601-607. 10.1051/jphyslet:019850046013060100 . jpa00232567

\section{HAL Id: jpa-00232567 https://hal.science/jpa-00232567}

Submitted on 1 Jan 1985

HAL is a multi-disciplinary open access archive for the deposit and dissemination of scientific research documents, whether they are published or not. The documents may come from teaching and research institutions in France or abroad, or from public or private research centers.
L'archive ouverte pluridisciplinaire HAL, est destinée au dépôt et à la diffusion de documents scientifiques de niveau recherche, publiés ou non, émanant des établissements d'enseignement et de recherche français ou étrangers, des laboratoires publics ou privés. 
Classification

Physics Abstracts

$61.50-81.30 \mathrm{~B}$

\title{
6-dimensional properties of $\mathbf{A l}_{0.86} \mathbf{M n}_{0.14}$ alloy
}

\author{
P. A. Kalugin, A. Yu. Kitayev and L. S. Levitov \\ L.D. Landau Institute for Theoretical Physics, U.S.S.R. Academy of Sciences, \\ 117334 Moscow V-334, U.S.S.R.
}

(Reçu le 16 avril 1985, accepté le 15 mai 1985)

\begin{abstract}
Résumé. - Les propriétés générales des phases présentant un groupe ponctuel icosaédrique et un ordre orientationnel à grande distance sont étudiées ici. On montre l'existence de six modes de Goldstone -3 phonons et 3 phasons. Un modèle pour la structure microscopique, cristalline dans un espace à six dimensions est proposé et les modes phasons sont discutés dans ce cadre. Des réseaux de Bravais sont déterminés et certains phénomènes physiques de AlMn dus à cette dimension six sont soulignés. Des arguments simples, du type de la théorie de Landau, montrent pourquoi la structure icosaédrique est favorisée.
\end{abstract}

\begin{abstract}
The general properties of the phases with the icosahedral point group and long-range orientational order are considered. 6 Goldstone modes -3 phonons and 3 phasons - are shown to exist. A model for the microscopic structure - a 6-D crystal - is proposed, and phason modes are discussed in this framework. Bravais lattice types are determined and some physical phenomena due to the peculiar AlMn dimension 6 are listed. Simple Landau-theory type arguments for advantages of icosahedral structure are put forward.
\end{abstract}

According to Plato's philosophy an icosahedron, as one of the regular Platonic solids, underpins the universe [1]. However its symmetry group turns out to be inconsistent with lattice translations [2] and unlike other Platonic solids, its symmetry group is not of common use in crystallography.

The remarkable Schechtman et al. experiments [3] changed this situation abruptly. The electron diffraction pattern of $\mathrm{Al}_{0.86} \mathrm{Mn}_{0.14}$ shows that it possesses an icosahedral point group and longrange positional order (an ability to scatter waves coherently).

Translational invariance is forbidden for $\mathrm{Al}_{0.86} \mathrm{Mn}_{0.14}$ so its microscopic structure is rather unusual. The first model for atomic arrangement was proposed by D. Levine and P. J. Steinhardt [5], who used R. Penrose's geometrical ideas [4]. The use of crystallography and, particularly, the Fourier transform of Penrose tiling was discussed by A. Mackay in [6]. Independently, another model - 6-dimensional crystal - was proposed in our paper [7]. (It is worth mentioning here that Penrose tiling is a particular case of our model and a simple generalization is necessary to construct a crystal having arbitrary forbidden space group [13].) 6 Goldstone modes - 3 acoustic and 3 "phason " - were shown to exist in $\mathrm{Al}_{0.86} \mathrm{Mn}_{0.14}$. Besides, a simple Landau-theory approach was used in [7] to discuss the probability of icosahedral phase formation at the firstorder transition when cooling rapidly from the melt. Per Bak $[9,10]$ has reached phenomenological conclusions similar to ours.

It should be mentioned that periodic structures in high dimensional spaces were already used 
to describe incommensurate systems (see, for example, Janner and Janssen [11]). But systems discussed up to now (" alchemist gold "- $\mathrm{Hg}_{3-8} \mathrm{AsF}_{6}, \mathrm{CDW}$, SDW) contain two weakly interacting subsystems having different lattice periods. This is not the case in $\mathrm{Al}_{\mathbf{0 . 8 6}} \mathbf{M n}_{\mathbf{0 . 1 4}}$, whose incommensurability is due to geometrical properties of point symmetry group but not to subsystems incommensurate with each other.

In the first part of the paper, the main results of [7] are briefly reviewed and possible 6-D Bravais lattices are described. Then in the second part, the physical properties of $\mathrm{Al}_{\mathbf{0 . 8 6}} \mathbf{M n}_{\mathbf{0 . 1 4}}$ due to its «6-dimensionality " are discussed.

\section{Crystal structure of $\mathrm{Al}_{0.86} \mathrm{Mn}_{0.14}$.}

We shall discuss here some general properties of icosahedral phase. Let the uniform liquid state be unstable with respect to icosahedral density wave formation :

$$
\delta \rho(x)=\rho_{0} \sum_{i=1}^{6} \cos \left(k_{0}\left(\mathbf{n}_{i} \mathbf{x}\right)+\alpha_{i}\right)
$$

( $\mathbf{n}_{i}$ are the unit vectors directed from the centre of an icosahedron to its vertices). Because of the interaction nonlinearity, waves of the form $\cos \left(k_{0}(\mathbf{N x})+\alpha\right)$ also appear $\left(\right.$ here $\mathbf{N}=\sum_{i=1}^{6} z_{i} \mathbf{n}_{i}$ with integer $z_{i}$ ). One checks easily that the $\left\{\mathbf{n}_{i}\right\}$ are linearly independent under the field of rational numbers. This is the reason why all $\alpha_{i}(i=1, \ldots, 6)$ are not fixed by nonlinear terms and the energy must be constant under all $\alpha_{i}$ transformations. So we have 3 additional " phason " modes corresponding to mutual displacements of icosahedral harmonics. Whether they are acoustic or diffusive depends on the structure of the system involved.

We propose a model to demonstrate how long-range order can appear from icosahedral neighbouring order relevant for metallic glasses.

Let us consider a 6-dimensional space $\left(\mathbb{R}^{6}\right)$ and an integer lattice having a space equal to $\sqrt{2}$ in it. We embed a 3-D subspace into it so that the projections of the lattice basic vectors $\left\{ \pm \mathbf{e}_{i}\right\}_{i=1, \ldots, 6}$ form vertices of icosahedron in $\mathbb{R}^{3}$. One checks immediately that $\left\{ \pm \mathbf{m}_{i}\right\}-$ the projections of $\left\{ \pm \mathbf{e}_{i}\right\}$ into $\mathbb{R}^{3 *}$ (the orthogonal complement to $\mathbb{R}^{3}$ ) - form 12 vertices of icosahedron too. Now we choose these integer points in the lattice that have their $\mathbb{R}^{3 *}$ projections into the icosahedron formed by $\left\{ \pm \frac{3}{2} \mathbf{m}_{i}\right\}_{i=1, \ldots, 6}$, i.e. we cut out a " tube " and consider all integer points in it. Now we project all these points into $\mathbb{R}^{3}$ to have the atom positions. (Note that no two of these projections are spaced at a distance less than 1.)

Although some of the icosahedral neighbours of an atom can be missed because of the « tube " cut off, every atom has at least 4 of them. To illustrate the existence of long-range positional order we calculate the Fourier transform of this structure. It is equal to the convolution of the lattice Fourier transform and the " tube » Fourier transform restricted on the reciprocal $\mathbb{R}^{3}$ space :

$$
\begin{aligned}
\rho_{k}=\int \rho(r) \mathrm{e}^{-i k r} \mathrm{~d}^{3} r & =(2 \pi)^{-3} \int\left[\left(\sum_{N} \delta\left(k-k_{N}\right)\right) *\left(\delta(k) F\left(k^{*}\right)\right)\right] \delta\left(k^{*}\right) \mathrm{d}^{3} k^{*}= \\
& =(2 \pi)^{-3} \sum_{N} \delta\left(k-k_{N}\right) F\left(k_{N}^{*}\right) ; \quad F\left(k^{*}\right)=\int \mathrm{e}^{-i k^{*} r^{*}} \theta_{\mathrm{T}}\left(r^{*}\right) \mathrm{d}^{3} r^{*}
\end{aligned}
$$

here $\mathbf{r}, \mathbf{k}, \mathbf{r}^{*}, \mathbf{k}^{*}$ are $\mathbb{R}^{3}$ and $\mathbb{R}^{3 *}$ compon nts of $\mathbf{R}$ and $\mathbf{K}$ respectively, $\mathbf{k}_{N}$ is the reciprocal $\mathbb{R}^{6}$ lattice vector, $\theta_{\mathrm{T}}\left(r^{*}\right)$ is equal to unity for the inner points of the tube and 0 for external ones.

Although the obtained Fourier transform is a system of $\delta$-functions densely filling the reciprocal space, almost all of their intensities $F\left(\mathbf{k}_{N}^{*}\right)$ are very small due to the « tube " cut off. 
One should mention that the nature of the 3 " phason " modes is obvious in the model considered. Phason transformations correspond to the tube displacement along $\mathbb{R}^{3 *}$ which yields atom hopping from one place to another. This is the reason for phasons to be diffusive, overdamped and maybe pinned.

As for $\mathrm{Al}$ and $\mathrm{Mn}$ atoms itself, their radii $\left(R_{\mathrm{Al}}=1.43 \AA, R_{\mathrm{Mn}}=1.30 \AA\right)$ allow to build an icosahedron of one $\mathrm{Mn}$ and $12 \mathrm{Al}$ atoms. This leads to a simple cubic 6-D lattice of $\mathrm{Mn}$ with Al atoms at the mid-points of the segments.

The computed electron diffraction pattern is given in the figures 1 to 5 . If one takes the possibility of higher order Born corrections into account and sensitivity of the pattern with respect to the form of a tube, then one concludes that correspondence with experimental results is good enough. (Unfortunately we calculated a low energy electron diffraction pattern in [7] but not a high energy one. This is the reason for divergence of [3] and [7] patterns. We thank D. S. Schechtman and his colleagues from Institute for Solid State Physics for the explanation of this fact.)

As for possible crystal symmetry types, they are determined by different 6-D space groups [13]. We present now some information about 5 Bravais lattices. 3 of them are invariant under all 6-D cube transformations : SC, FCC (integer points with even sum of coordinates) and BCC

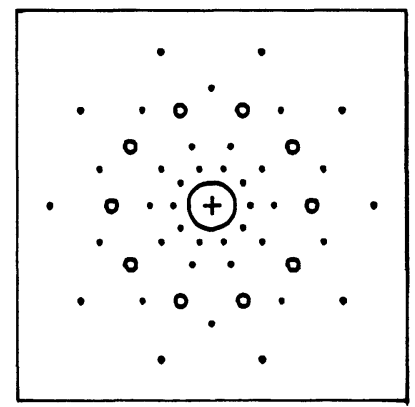

1

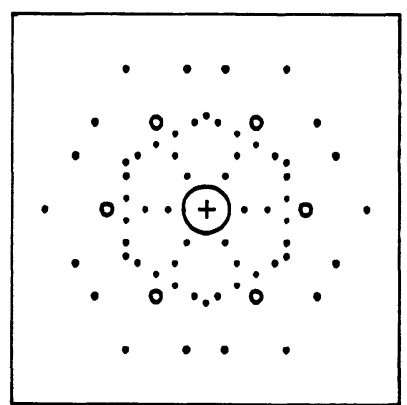

2

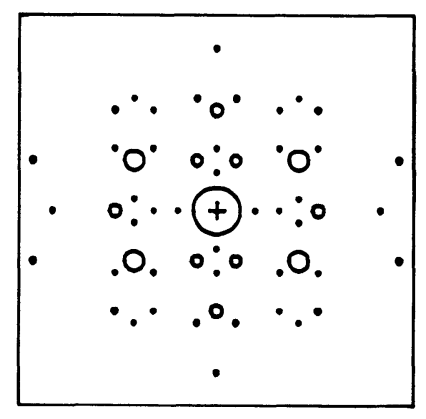

3

Figs. 1, 2, 3. - Computed diffraction pattern for 5-, 3- and 2-fold axis respectively. The " tube " form factor $F\left(\left|k^{*}\right|\right)$ is assumed to have a form : $\left(\left|k^{*}\right| R\right)^{-3}\left(\sin \left(\left|k^{*}\right| R\right)-\left|k^{*}\right| R \cos \left(\left|k^{*}\right| R\right)\right), R=1.2$. Only peaks above some given intensity are shown.

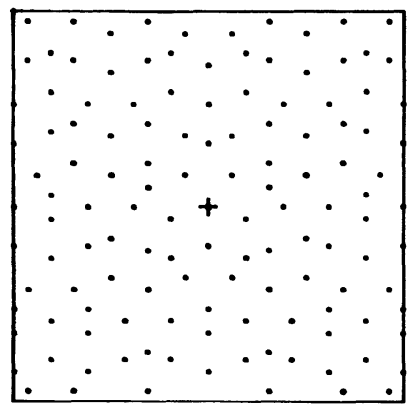

4

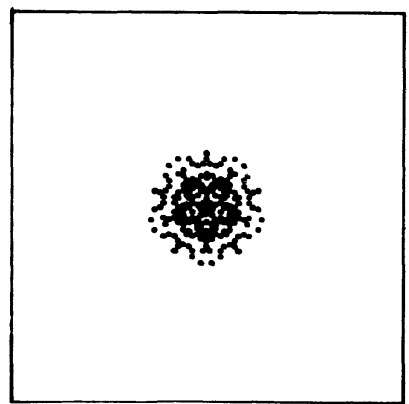

5

Figs. 4, 5. - Evidence for Penrose tiling to be a kind of a " tube ». Fig. 4 : Penrose tiling points of the 4th generation, Fig. 5 : the same points after Galois permutation of basic vectors : $z_{1} e_{1}+z_{2} e_{2}+z_{3} e_{3}+$ $z_{4} e_{4}+z_{5} e_{5} \rightarrow z_{1} e_{1}+z_{2} e_{4}+z_{3} e_{2}+z_{4} e_{5}+z_{5} e_{3}$. (It corresponds to the projection into the orthogonal complement.) 
(integer points having one and the same parity of coordinates). In addition to these, two other lattices exist. One of them is generated by vectors of $T_{\mathrm{g}}(x)$ type (here $\mathrm{g}$ - all icosahedral) transformations and $x=(1,1,1,1,1,1)$, and the second is given by an analogous procedure using $x^{\prime}=(1,1,1,1,1,-1)$.

These two lattices have the icosahedral point-group, which is a subgroup of a 6-D cubic group. It is interesting that no small deformation of 3 high symmetry lattices lead to the 2 lower symmetry ones. This situation is analogous to a well-known crystallographical exceptional fact : the rhombohedral class space group may have both rhombohedral and hexagonal Bravais lattices [2]. But it is worth mentioning that SC lattice fits experimental data best of all.

To take into account the atomic interaction let us replace the « tube " on $\mathbb{R}^{3}$ and atoms on 3-D pancakes so that every pancake looks like an icosahedron lying along $\mathbb{R}^{3 *}$. The equivalence of such a model to the considered one is obvious. The interatomic interaction deforms the pancakes and maybe tears them but leaves their arrangement periodic. So the remarkable diffraction pattern is destroyed by this interaction.

Now we are going to put forward some phenomenological Landau-theory type arguments of icosahedral phase formation at a rapid melt cooling with 1-order transition. According to [8] we expand the free energy in terms of order parameter $\rho_{q}$ in the following way :

$$
\begin{aligned}
\varphi[\rho] & =\varphi_{2}+\varphi_{3}+\cdots \\
\varphi_{2} & =\sum_{q} A(|\mathbf{q}|) \rho_{q} \rho_{-q} \\
\varphi_{3} & =\sum_{q_{1} q_{2}} B\left(\mathbf{q}_{1}, \mathbf{q}_{2}\right) \rho_{q_{1}} \rho_{q_{2}} \rho_{-q_{1}-q_{2}}
\end{aligned}
$$

and calculate the value $C=\left|\varphi_{3}\right| / \varphi_{2}^{3 / 2}$ describing the height of a potential barrier which the system surmounts during the crystallization.

Let us assume for the sake of simplicity that $B\left(q_{1}, q_{2}\right)=B_{0}, A(|q|)=A_{0}+A_{1}\left(|q| / k_{0}-1\right)^{2}$. Then according to [8] one would take the density wave as $\rho_{0} \sum_{i} \cos \left(k_{0}\left(\mathbf{n}_{i} \mathbf{x}\right)+\alpha_{i}\right)$, where $n_{i}$ is the set of unit vectors with necessary point group. Thus it is implied that higher order harmonics arise due to nonlinear effects only. If $A_{0} \simeq A_{1}$, this is the case only for wave vectors that are not too close to $k_{0} n_{i}$. For icosahedral symmetry this condition is violated for second harmonics already (the edge of an icosahedron is only $5 \%$ longer than the distance from its centre to the vertex). Therefore the conclusion [8] that the BCC structure is more likely than the icosahedral one seems to be not very convincing. Taking into account 1- and 2-order harmonics by

$$
\delta \rho(x)=\rho_{0}\left(\lambda_{1} \sum_{i=1}^{6} \cos \left(k\left(\mathbf{n}_{i} \mathbf{x}\right)\right)+\lambda_{2} \sum_{i, j=1}^{6} \cos \left(k\left(\left(\mathbf{n}_{i}-\mathbf{n}_{j}\right) \mathbf{x}\right)\right)\right)
$$

( $i$ and $j$ are the numbers of the neighbouring vertices of an icosahedron) gives rise to

$$
C_{\mathrm{ICO}}<C_{\mathrm{BCC}} \quad \text { if } \quad A_{1} \lesssim 70 A_{0} .
$$

The icosahedral phase forming condition is rather weak. Hence, although this phase is metastable [3] its formation during rapid cooling is very likely sometimes.

\section{6-dimensional properties.}

The possibility of "phason " motion is an interesting and difficult question. Strong incommensurability, absence of two weakly interacting systems, necessity of atoms hopping yields considerable damping. Moreover pinning of these modes by impurities is possible. Ignoring 
here all these questions and supposing the " phason » dynamics to be diffusive we shall describe some properties of Al-Mn type substances caused by phasons.

Let us revise some necessary mathematical facts concerning the icosahedral point-group. It has 5 irreducible representations $1,3, \overline{3}, 4,5$, where the number indicates the dimensionality. (Full character table can be found in [10] and [13].)

3 is a standard representation with matrix elements of the form $a+b \sqrt{5}(a, b$ - rational numbers), $\overline{3}$ differs from 3 by a sign before $\sqrt{5}, 5$ is the representation in the group $A_{5}$ of even permutations of 5 elements. Necessary expansions of representation products are given by :

$$
\begin{array}{ll}
3 \cdot 3=1+3+5 & 3 \cdot \overline{3}=4+5 \\
\overline{3} \cdot \overline{3}=1+\overline{3}+5 & 3 \cdot 5=3+\overline{3}+4+5 .
\end{array}
$$

Simple analysis shows that the action of the icosahedral group under six phases $\alpha_{i}$ has two stable subspaces : phonons $u_{i}(i=1, \ldots, 3)$ - representation 3 and phasons $v_{\alpha}(\alpha=1, \ldots, 3)$ representation $\overline{3}$. The expansion of the elastic part of free energy in terms of $u_{i j}, \partial_{i} v_{\alpha}$ in quadratic approximation takes the form

$$
F(u, v)=A_{i j k l} u_{i j} u_{k l}+B_{i j k, \alpha} u_{i j} \partial_{k} v_{\alpha}+C_{i j, \alpha \beta} \partial_{i} v_{\alpha} \partial_{j} v_{\beta}
$$

with symmetry restrictions on $A, B$ and $C$ :

$$
\begin{gathered}
A_{i j k l}=\frac{\lambda}{2} \delta_{i j} \delta_{k l}+\mu \delta_{i k} \delta_{j l}, \quad C_{i j, \alpha \beta}=a \delta_{i j} \delta_{\alpha \beta}+c d_{i j, \alpha \beta}, \quad B_{i j k, \alpha}=b t_{i j k}, \\
d_{i i, \alpha \beta}=d_{i j, \alpha \alpha}=0, \quad t_{i i k, \alpha}=t_{i k i, \alpha}=t_{k i i, \alpha}=0
\end{gathered}
$$

One should mention the phason-phonon mixing term in (7) which leads to a phason response to elastic deformation :

$$
\begin{gathered}
\partial_{i} v_{\alpha}=\Lambda t_{i j k, \alpha} u_{j k} \\
\Lambda=-\frac{b}{2(a+x c)}, \quad \kappa=\frac{t_{i j k, \alpha} d_{k l, \alpha \beta} t_{i j l, \beta}}{t_{m n p, \lambda} t_{m n p, \lambda}}
\end{gathered}
$$

(if $u_{i j}$ is an isotropic deformation $\partial_{i} v_{\alpha}$ vanishes).

Phason deformation alters the diffraction pattern significantly. To explain it we use the « tube » model. Phason deformation leads to the displacement of the tube and simple calculation shows that peak intensities remain the same while their displacements change :

$$
\delta q_{i}=u_{i j} q_{j}+\Lambda t_{i j k, \alpha} u_{j k} q_{\alpha}^{*}
$$

(here $q_{i}$ and $q_{\alpha}^{*}$ are $\mathbb{R}^{3}$ and $\mathbb{R}^{3 *}$ coordinates respectively). The first term describes the usual effect of deformation influence on a diffraction pattern, and the second the effect we are interested in. This displacement is the more intense the greater $\left|q^{*}\right|$ is. So far as it is possible to find the peak with arbitrary $\left|q^{*}\right|$ in any region of the pattern (maybe with rather weak intensity) the displacements should be visible even for weak deformations.

Peculiarities of phason dynamics may give rise to strong delay of the diffraction pattern phason response to deformation.

The Debye-Waller factor changes abruptly and for the peak coordinates $\left(\mathbf{q}, \mathbf{q}^{*}\right)$ is given by

$$
D_{q, q^{*}}=\left\langle\mathrm{e}^{i\left((q u(x))+\left(q^{*} v(x)\right)\right)}\right\rangle^{2}=\exp \left(-q^{2} \frac{\left\langle u^{2}\right\rangle}{3}-\left(q^{*}\right)^{2} \frac{\left\langle v^{2}\right\rangle}{3}\right) .
$$


The average in (11) is made over long wavelength phonons and phasons. The $D_{q, q^{*}}$ dependence on $q^{*}$ is unusual and it gives rise to great attenuation for weak peaks. It seems to be interesting to explore the $\left\langle v^{2}\right\rangle$ temperature dependence experimentally.

The phonon-phason interaction changes the phonon spectrum which is isotropic if this interaction can be neglected or if the phasons are pinned. But if phasons take part in dynamics, the phonon spectrum becomes anisotropic. If we adopt the phason dynamics to be guided by simple diffusive equation :

$$
\gamma \partial_{t} v_{\alpha}=-\frac{\delta F}{\delta v_{\alpha}}
$$

then for $\omega \ll \gamma$ we will have an equation :

$$
\begin{gathered}
\omega^{2} u_{i}=\frac{\lambda}{2} K_{i}(K u)+\mu K^{2} u_{i}+i \frac{1}{\omega|K|} S_{i j k l m n} K_{j} K_{k} K_{l} K_{m} K_{n} \\
S_{i j k l m n}=B_{i j k, \alpha} B_{l m n, \alpha}
\end{gathered}
$$

$3^{6}=16.1+\cdots$, but $\mathrm{SO}(3)$ has only 15 6-degree invariants so $S_{i j k l m n}$ necessarily includes anisotropic part (one may distinguish it if one demands all traces to be equal to 0 ).

This is the reason for low frequency phonons to differ from those in an isotropic solid even in first $\frac{\omega}{\gamma}$ approximation. The separation of two transverse modes becomes unambiguous. These two modes are eigen vectors of the tensor $T_{i j}$ :

$$
T_{i j}=P_{i i^{\prime}} P_{j j^{\prime}} S_{i^{\prime} j^{\prime} k l m n} n_{k} n_{l} n_{m} n_{n}, \quad P_{i i^{\prime}}=\delta_{i i^{\prime}}-n_{i} n_{i^{\prime}}
$$

(where $n_{i}$ is the unit vector in the sound propagation direction). The anisotropic part of the longitudinal mode attenuation is proportional to $S_{i j k l m n} n_{i} n_{j} n_{k} n_{l} n_{m} n_{n}$.

There are some peculiarities of AlMn dislocation structure, 6 dislocation types exist instead of usual 3 (we are grateful to G. E. Volovik, who told us about it). Equation (7) shows that the dislocation line energy diverges logarithmically with coefficient before $\ln \frac{L}{a}$ depending on all 6 components of the Burgers vector (for example it is proportional to $b^{2}+b^{* 2}$ if $\lambda=b=c=0$, $a=\mu$, here $\mathbf{b}$ and $\mathbf{b}^{*}$ are $\mathbb{R}^{3}$ and $\mathbb{R}^{3 *}$ components of the Burgers vector). So as in usual crystals, dislocation loops would appear and which of them are advantageous depends on 6-D Burgers vector length.

In a similar manner the rules determining the growth velocity of crystal faces change. By analogy to [12] we conclude that the faces having small 6-D Miller indices will grow slower and have greater area.

\section{Concluding remarks.}

The peculiar 6-dimensionality of $\mathrm{Al}_{0.86} \mathrm{Mn}_{0.14}$ should manifest itself in some observable physical effects discussed above. But because of small grain size in [3], not all of them are easy to observe. We list now the most promising ones.

We have discussed already the phason-phonon response which leads to unusual sensitivity of the diffraction pattern to the external deformation. For example, if 3 maxima are collinear before the deformation, they should displace so that their collinearity is destroyed. But because we do not know phason diffusion coefficient we can not evaluate the equilibrium establishment time. However if it is too great, another interesting effect may be observed : phason deformations 
will hinder dislocations from motion and reveal themselves in anomalous viscosity. Then high density of relaxation states may lead to anomalously great $f^{-1}$-noise.

\section{Acknowledgments.}

V. L. Pokrovsky and L. P. Gor'kov provided stimulation, advise and encouragement at all stages. We are grateful to G. E. Volovik, S. E. Burkov and Per Bak for interesting and sometimes useful discussions.

Recent independent works were cited by referees and are acknowledged by the authors. On six component dislocations : LeVINe, D., Lubensky, T. C., Ostland, S., Ramaswamy, S., SteInhardt, P. J. and TONER, J., Phys. Rev. Lett. 54 (1985) 1520. On projection from 6D : ElSER, V., Phys. Rev. Lett. 54 (1985) 1730, and to be published ; DunEaU, M. and KatZ, A., Workshop on Mathematical Crystallography, Institut des Hautes Etudes Scientifiques, L. Michel and M. Senechal, edts., January 1985, and submitted to Phys. Rev. Lett. In this last publication, the size of the tube is somewhat larger.

\section{References}

[1] Plato Works v. 6 (Moscow, Russian, 1879), p. 433.

[2] Landau, L. D. and Lifshitz, E. M., Statistical Physics, 2nd ed. (Pergamon, New York, 1968), part 1, pp. $130,131$.

[3] Schechtman, D., Blech, I., Gratias, D. and Cahn, J. W., Phys. Rev. Lett. 53 (1984) 1951.

[4] Penrose, R., Bull. Inst. Maths Its Appl. 10, No 7/8 (1974) 266.

For a popular article on Penrose tiling see : Gardner, M., Sci. Am. 236 (1977) 110.

[5] Levine, D., Steinhardt, P. J., Phys. Rev. Lett. 53 (1984) 2477.

[6] Mackay, A. L., Physica A 114 (1982) 609.

[7] Kalugin, P. A., Kitayev, A. Yu., Levitov, L. S., Pis'ma v ŽETF 41 (1985) 119.

[8] Alexander, S. and Mc Tague, J. P., Phys. Rev. Lett. 41 (1978) 702.

[9] BaK, Per, Phys. Rev. Lett., submitted.

[10] BaK, Per, Phys. Rev., submitted.

[11] JANNer, A. and Janssen, T., Acta Crystallogr., Sect. A 36 (1980) 408.

[12] Janner, A. and Janssen, T., Phys. Rev. Lett. 45 (1980) 1700.

[13] Kalugin, P. A., KitaYev, A. Yu., Levitov, L. S., $\check{Z} E T F$, to be published. 